\title{
Pap Smear Combined with HPV Testing: A Reasonable Tool for Women with High-grade Cervical Intraepithelial Neoplasia Treated by LEEP
}

\author{
Ling-Zhao Mo ${ }^{\circledR}$, Hong-Lin Song ${ }^{\circledR}$, Jian-Li Wang, Qing He, Zhang-Can Qiu, Fei Li*
}

\begin{abstract}
Background: To evaluate HPV testing by Hybrid Capture II (HCII) in conjunction with cytology in detecting the residual/recurrence disease after treatment of high-grade cervical intraepithelial neoplasia (CIN II-III) with loop electrosurgical excision procedure (LEEP). Materials and Methods: A retrospective review of 158 patients with histologically confirmed CIN II-III who underwent LEEP between January 2011 and October 2012 was conducted. Post-treatment control was scheduled at the 3rd, 6th, 12th and 18th month. All patients were followed up by Pap smear and HR-HPV genotype and viral load testing. Results: Pre-treatment, HR-HPV DNA, was detected in all specimens of the patients. At follow-up, 25 patients were diagnosed as the residual/recurrent disease during the FU visit, among whom, 16 patients with positive margin: 13 patients (52\%) with HR-HPV DNA+/ cytology+, 2 patients $(8 \%)$ with HR-HPV DNA+/cytology-, 1 patient (4\%) with cytology+/ HR-HPV DNA-; 9 patients with clean margin - 5 patients $(55.6 \%)$ with HR-HPV DNA+/cytology+; 2 patients $(22.2 \%)$ with HRHPV DNA+/cytology-, 2 patients $(\mathbf{2 2 . 2 \%})$ with cytology+/ HR-HPV DNA-. None of them persisting HR-HPV DNA-/cytology- with positive or negative margin was identified as the residual/recurrent disease. The majority of residual/recurrent disease was detected at the 12th and 18th month $F U$, and there was almost no difference in the sensitivity and negative predictive value (NPV) between at the 3rd month and the 6th month FU visits. 14 residual/recurrence disease (14/46:30.4\%) had pre-treatment high viral load (>5 000 RUL/PC) and 11 (11/112, 9.8\%) with pre-treatment low viral load, $P<0.05$. Conclusions: (1) The persistence HR-HPV DNA is the root cause of the residual/recurrent disease for the women treated for high-grade CIN; the pre-treatment viral load and margin can be seen as the predictor. (2) The FU visit beginning at the 6th month post-treatment and lasting at least 24 months with the combination of cytology and HPV testing. (3) Patients with high pre-treatment HPV load, which is considered as one risk of developing the residual/recurrent disease, should be paid more attention (especially above 500RUL/PC) to by clinicians.
\end{abstract}

Keywords: The residual/recurrent disease - positive/negative margin - persistence HR-HPV DNA - viral load - follow-up

Asian Pac J Cancer Prev, 16 (10), 4297-4302

\section{Introduction}

The high-grade cervical intraepithelial neoplasia (CIN II-III) is considered as high risk for cervical invasive carcinoma if it is left untreated (Pinto et al, 2000). Based on efficacy evaluation (Prendiville et al., 1989; Paraskevaidis et al., 2001), the loop electrosurgical excision procedure (LEEP) has become a popular choice for the treatment of high-grade cervical intraepithelial neoplasia (CINII-III) at present. One of the benefits of LEEP is that it provides the pathologist with an intact specimen to study an intraepithelial or superficially invasive lesion in its entirety. However, even though this method has gained a great success on cervical conization for CIN II-III treatment, the recurrence rate still ranges from 2\% 38\% (Gardiel, et al., 1997; Murdoch et al., 1992;
Hanau et al., 1997) in patients with clean margins.

Follow-up (FU) with cytology and colposcopic evaluation of the cervix, the preferred strategy for PostLeep CIN II-III control at present, which is traditionally performed with repeated colposcopic examination and cervical cytology, but it ought to be kept in mind that the Pap smear test might be negative even in the presence of biopsy-confirmed CIN II-III (Flannelly et al., 2001; Tangtrakul et al., 2002).

Human papillomavirus (HPV), the most common causation of sexual transmitted infections (STDs) in the United States (Hoskins et al., 1992), had been considered as an etiologic agent for cervical cancer since 1977 (Meisels et al., 1977) and been currently unequivocally linked with the development of precancerous lesions of the cervix, especially, high-risk HPV (HR-HPV) are closely 
associated with the presence of high-grade squamous intraepithelial lesions (HISIL) or CIN II-III (Baron et al., 1970; Dalstein et al., 2004). An increasing interest has been focused on HPV detection techniques proposed in the treatment and follow-up of patients with CIN II-III. Indeed, residual or recurrent disease only occurs in women with persistent HPV infection after treatment (Nagai et al., 2000; Nobbenhuis et al., 2001). Thus growing evidences have indicated that HPV testing is useful as an adjunct to cervical cytology in detecting women with increasing risk of persistence or recurrence of CIN II-III following cervical conization (Zielinski et al., 2003; Houffin et al., 2003; Bar-Am et al., 2003; Costa et al., 2003).

The aim of this retrospective study is to evaluate HPV testing by Hybrid Capture II (HCII) in conjunction with cytology in detecting the residual/recurrent disease after treatment of high-grade cervical intraepithelial neoplasia (CIN II-III) with Loop electrosurgical excision procedure (LEEP).

\section{Materials and Methods}

\section{General data}

A retrospective analysis was performed on 158 patients with histologically conformed CINII and CIN III who were treated by LEEP in the Department of Gynecological Oncology of Tumor Hospital Affiliated to Guangxi Medical University from January 2011 to October 2012. The inclusion criteria was CIN II or III confirmed in the cervical conization specimens, and all patients fulfilled at least three follow-up visits at the 3rd, 6th and 12th month after treatment. The exclusion criteria consisted of pregnancy, clinical signs of immunosuppression, and the positive HIV test. The patients were excluded without all the follow-up visits, leaving 158 patients enrolled in this study.

\section{Follow-up routine}

At the pretreatment visit, all women were submitted to an interview concerning the clinical, social, and demographic data. After the interview, a complete gynecological examination was performed, with collection of endocervical specimens for cervical cytology and HCII, followed by colposcopic examination of the cervix.

Follow-up (FU) visit consisted of examining the patients at the $3 \mathrm{rd}, 6 \mathrm{th}, 12 \mathrm{th}$ and 18 th month after cervical conization and thereafter kept under annual surveillance after this period, until the last two HPV testing/Pap smears were normal. This routine included the patient's interview and the complete gynecological examination, with collection of HCII and Pap smear test samples. Colposcopy examination was performed with either persistent positive HPV DNA or abnormal Pap smears ( $\geq$ atypical squamous cells undetermined significance, ASCUS). Patients presenting with HISIL smear or abnormal pattern on colposcopy underwent a new excision procedure, biopsy, LEEP or hysterectomy.

\section{Pap smear and HPV testing}

In every FU visit, women underwent a pelvic examination, and 2 samples of exfoliated cells were obtained from the cervix: the first sample was collected with a Cytobrush ${ }^{\circledR}$ Plus (Medscand medical, Malmö, Sweden) for conventional cytology and the second was collected with the Digene Cervical Sampler ${ }^{\circledR}$ (Digene, Gaithersburg, MD) for HPV testing, place in vial containing $1 \mathrm{~mL}$ Digene specimen Transport Medium ${ }^{\circledR}$ and stored at $-20^{\circ} \mathrm{C}$ until proceed.

Final cytological diagnoses were classified according to the Bethesda system as normal or inflammatory, ASCUS, low-grade squamous intraepithelial lesion (LGSIL), or high-grade squamous intraepithelial lesion (HGSIL).

HCII HR- HPV testing was performed with the Hybrid Capture II (HCII) system (Digene) according to the manufacture's instruction, using the specific HPV RNA probe B for carcinogenic high-risk HPV test (HR-HPV) $16,18,31,33,35,39,45,51,52,56,58,59$ and 68 . The presence or absence of HPV DNA in the specimen was defined according to the strength in relative light units (RLU) compared with $1 \mathrm{pg} / \mathrm{mL}$ HPV16 DNA-positive control (PC). The sample was considered positive when the ratio of RLU/PC was $\geq 1$. Values provide an approximate determination of the global HR-HPV DNA load.

\section{Loop electrosurgical procedure and histology}

The goal of LEEP was to remove the lesion and the transformation zone as a one-piece cone specimen in its entirety. All cervical conization procedure was performed under the condition that the cervix was exposed by an adapted speculum allowing smoke to evacuate. Secondary to local anesthesia in each quadrant, the abnormal epithelium was delineated with Ligol's iodine, The whole surgical procedure was carried out with the wire loop selected according to the size of the area to be excised. The base of the wound was cauterized with a ball electrode. The 12 o'clock position in the excised specimen was marked by suture. The removed tissue was fixed with $10 \%$ phosphate-buffered formalin and submitted to histopathologic examination after then. Dysplasia lesions were analyzed according to the WHO's criteria's (Valasoulis et al., 2014) and classified as negative, CIN I, CIN II, CIN III severe dysplasia or carcinoma in situ, or microinvasive carcinoma.

\section{Statistical data analysis}

The sensitivity, specificity, positive and negative predictive values (PPV and NPV) were calculated for evaluating the efficacy of the post-LEEP detection tools as HCII, Pap smear, and the combination of both tests for the diagnosis of residual/recurrent disease. Fisher's exact test calculates for the independence and the trend to identify the potential risk factors of residual/recurrent disease as HR-HPV load, the cone margin, cytology and age.

\section{Results}

\section{Characteristics of epidemiology}

The median age of the total patients with histologically confirmed CIN II-III at the time of the treatment, is 35.12 years old (range 19 57). Table 1 showed the significant 
Table 1. Baseline Data Between No Residual/Recurrence Lesion and Residual/Recurrence Lesion [n(\%)]

\begin{tabular}{|c|c|c|c|c|}
\hline Characteristics & $\mathrm{n}$ & $\begin{array}{c}\text { No residual /recurrence } \\
\text { lesion }[\mathrm{n}(\%)]\end{array}$ & $\begin{array}{l}\text { Residual /recurrence } \\
\text { lesion }[\mathrm{n}(\%)]\end{array}$ & $P$ \\
\hline Age & $35.12(19 \sim 57)$ & $35.26(19 \sim 57)$ & $34.68(22 \sim 53)$ & \\
\hline \multicolumn{5}{|c|}{ Cytology before conisation } \\
\hline Normal & 1 & 1 & 0 & \\
\hline Asu-H & 1 & 1 & 0 & \\
\hline ASCUS & 18 & $17(94.6)$ & $1(5.6)$ & \\
\hline LSIL & 31 & $27(87.1)$ & $4(12.9)$ & \\
\hline HISIL & 107 & $87(81.3)$ & $20(18.7)$ & \\
\hline \multicolumn{5}{|c|}{ Histology of conisation } \\
\hline CINI & 0 & 0 & & \\
\hline CINII & 45 & $37(82.2)$ & $8(17.8)$ & \\
\hline CINIII & 69 & $64(92.8)$ & $5(7.2)$ & \\
\hline Cacinoma in situ & 44 & $32(72.7)$ & $12(27.3)$ & \\
\hline \multicolumn{5}{|l|}{ Cone margins } \\
\hline Negative & 117 & $108(92.3)$ & $9(7.7)$ & $<0.001^{\mathrm{a}}$ \\
\hline Positive & 41 & $25(61)$ & $16(39)$ & \\
\hline \multicolumn{5}{|c|}{ HPV DNA before conisation } \\
\hline Negative & 0 & 0 & 0 & $<0.05^{\mathrm{a}}$ \\
\hline $1-100$ & 52 & $48(92.4)$ & $4(7.6)$ & \\
\hline $100-500$ & 60 & $53(88.3)$ & $7(11.7)$ & \\
\hline $500-1000$ & 21 & $16(76.2)$ & $5(33.8)$ & \\
\hline$>1000$ & 25 & $16(64)$ & $9(46)$ & \\
\hline \multicolumn{5}{|c|}{ HPV DNA after conization } \\
\hline Negative & 136 & $133(97.8)$ & $3(2.2)$ & \\
\hline $1-100$ & 14 & 0 & $14(100)$ & \\
\hline $100-500$ & 7 & 0 & $7(100)$ & \\
\hline$>500$ & 1 & 0 & $1(100)$ & \\
\hline
\end{tabular}

${ }^{a}$ Fisher's exact test

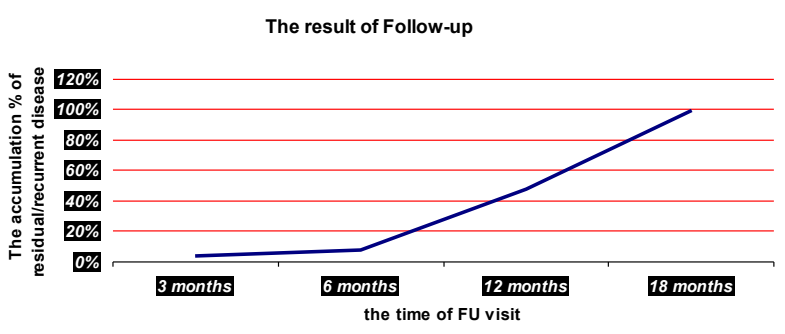

Figure 1. [please supply]

association between the pre-treatment high viral load and the positive cone margin with the residual/recurrent disease. During the follow up, 25 women $(15.8 \%$ of the total) showed residual or recurrent disease. The median age of these women was 34.68 years old (range 22 53), however, the median age of the non-recurrent case was 35.26 years old (range from 19 57), $P=\mathrm{NS}$. HR-HPV DNA was positive in all cases in the pre-treatment assay: among 112 women (70.9\%) with viral load inferior to 500 RLU/PC, 11 (9.8\%) showed consistent HR-HPV infection and developed into the residual/recurrent diseases during at least 6 months after treatment; while 46 women (29.1\%) with viral load superior to 500 RLU/PC, 14 (30.4\%) showed consistent HR-HPV infection and developed into the residual/recurrent diseases during a least 6 months after treatment, $(P<0.05)$. The geometric mean pre-treatment HR-HPV load in RLU in the group of residual/recurrent case was $(994.1 \pm 1117.5)$ and $(365 \pm 539.3)(p=0.014)$ in the group of non-recurrent. The total surgical positive/ negative margin cases were 41 (25.9\%) / 117 (74.1\%),
Table 2. Correlation of HPV Testing with Residual Disease in Women with Cone Margin

\begin{tabular}{lcrrr}
\hline $\begin{array}{l}\text { All patient } \\
(\mathrm{n}=158)\end{array}$ & $\begin{array}{c}\text { Pre- } \\
\text { treatment }\end{array}$ & $\begin{array}{c}\text { Pro- } \\
\text { treatment }\end{array}$ & $\begin{array}{c}\text { Residual } \\
\text { desease }\end{array}$ & $P$ \\
\hline Positive cone margin $(\mathrm{n}=41)$ & & & \\
HPV+/cytology + & 41 & 13 & 13 & \\
HPV+/cytology - & 0 & 2 & 2 & \\
HPV-/cytology+ & 0 & 1 & 1 & \\
HPV-/cytology- & 0 & 25 & 0 & $<0.0001^{\text {a }}$ \\
Negative cone margin $(\mathrm{n}=117)$ & & & \\
HPV+/cytology + & 117 & 5 & 5 & \\
HPV+/cytology- & 0 & 2 & 2 & \\
HPV-/cytology+ & 0 & 2 & 2 & \\
HPV-/negative- & 0 & 108 & 0 & $<0.0001^{\text {a }}$ \\
\hline
\end{tabular}

a Fisher's exact test

respectively. 9 women $(9 / 25,36 \%)$ with negative margin were identified as the residual/recurrent diseases at the end of FU, while in the group with positive margin, $16(16 / 25$, $64 \%)$ showed the same case, $(P<0.001)$.

\section{Follow-up post-treatment}

Figure 1 showed the results of all women in FU according to the cytology/HPV status at the time indicated. At the 3rd and 6th month of FU visits, only 2 women (2/25, $8 \%$ ) with cytology/HR-HPV positive were confirmed as CIN II by histology. The other 23 women $(23 / 25,92 \%)$ with HR-HPV positive and/or abnormal Pap smear were identified as the residual/recurrent disease after the $6^{\text {th }}$ month FU visit. Compared with the sensitivity, the specificity, NPV and PPV, at the beginning of the two FU visits, there was not essential distinction between these 
Table 3. Persistence of HPV DNA in Postconization with CIN 2-3 of the Cervix

\begin{tabular}{|c|c|c|c|c|c|}
\hline \multicolumn{2}{|c|}{ HPV DNA } & \multirow[t]{2}{*}{ No. Of patient (\%) } & \multicolumn{2}{|c|}{ Cytology (pro-treatment) } & \multirow[t]{2}{*}{$P$} \\
\hline Pre-treatment & Pro-treatment & & Abnormal & Normal & \\
\hline Positive & positive & $22(14 \%)$ & 18 & 4 & $<0.0001^{\mathrm{a}}$ \\
\hline Negative & Negative & $136(84 \%)$ & 3 & 133 & \\
\hline Total & 158 & & & & \\
\hline
\end{tabular}

a Fisher's exact test

two FU visits. On the other hand, with the FU visit going on, the results of HCII were gradually getting better than those of cytology, even better than those of the combining two assays.

\section{Identification of predictors of residual/recurrent disease}

Table 2 showed the correlation between HPV testing /cytology and residual/recurrent disease in cone margin of the patients. HR-HPV DNA was detected in all the 158 study objects before cervical conization. After treatment, in the group of the positive margin, the persistence of HR-HPV DNA +/cytology+ occured in 13 cases (13/41, $31.7 \%)$, HR-HPV DNA+/cytology- in 2 case $(2 / 41,4.9 \%)$ and one case $(1 / 41,2.4 \%)$ for cytology+/HR-HPV DDA-, which were identified as the residual/recurrent disease during FU visit; among 25 cases $(25 / 41,61 \%)$ with HRHPV DNA-/cytology-, none of them was diagnosed as the residual/relapse disease during FU visit $(P<0.0001)$. In the group of negative margin, The HPV test was persistently positive in 4 cases $(4 / 117,3.4 \%)$ with cytology positive and in 2 cases $(2 / 117,1.7 \%)$ with cytology negative; 2 cases $(2 / 117,1.7 \%)$ with cytology positive and HR-HPV DNA negative, which also were diagnosed as the residual/ recurrent disease during FU visit, however, none of the 108 (108/117, 92.3\%) with cytology negative and HR-HPV DNA negative was identified as the residual/recurrent disease during FU $(P<0.0001)$.

Among 22 women with the persistence positive HR-HPV DNA after treatment, 18 (18/22, 81.8\%) with cytology positive turned abnormal of the cervix again and $4(4 / 22,18.2 \%)$ with normal cytology during FU visit, who were confirmed abnormal histology. 3 women (3/136, $2.2 \%$ ) with HR-HPV DNA negative were found abnormal of the cervix after treatment, who were diagnosed as the residual/recurrent disease during FU visit, while none of 133 women $(133 / 136,97.8 \%)$ persisting HR-HPV DNA-/ cytology- after treatment showed the residual/recurrent disease during the FU, $(P<0.0001)$ (Table 3$)$.

\section{Discussion}

The majority of epidemiological studies have confirmed a very appreciable increase in the incidence and prevalence of CIN all over the world. However, the median age of women diagnosed CIN shows a decreasing trend, indicating that the HR-HPV is infecting women in the early days. The previous studies have revealed the result coincident with the augmentation of cervical cancer among the young women before 40 years old in certain countries (Quinn et al., 1999. Li et al., 2004; Zhang et al., 2014; Wang et al., 2014; Shen et al., 2014; Du et al., 2014). The median age of the total patients with histologically confirmed CIN II-III at the time of the treatment is 35.12 years old (range 19- 57), 44 women among them were diagnosed as cervical carcinoma in situ with 36.12 years old as the median age; moreover, there was no significant statistic difference at the median age between women with residual/recurrent case (34.68 years old) and non recurrent cases ( 35.26 years old). The study result has indicated that cognition, earlier diagnosis and treatment in clinical stage are the key points for the control of the CIN which will probably develop into the cervical cancer.

In the present study, HCII in conjunction with cytology is used for monitoring the objects after being treated for CIN II-III, the reasons for which include: (1) pap smear test has the inherent flaws, particularly its relatively high false negative rate (Nobbenhuis et al., 2001); (2) the goal of FU is not to miss active disease, therefore the sensitivity and negative predictive value (NPV) are valued higher than the specificity and false positive test. HPV testing has high sensitivity and negative predictive value (NPV) for detecting residual/recurrent diseases as a good index of disease clearance showing in several studies (Zielinski et al., 2003; Costa et al., 2003; Sarian et al., 2004). The result in the present study demonstrated that: the results of cytology were better than those of HCII based on comparison of sensitivity and specificity, PPV and NPV at the beginning of the two groups of FU visits; the results of combining the two assays reached to $100 \%$. Nevertheless, in the two final FU visits, the more residual/recurrent diseases were diagnosed, the more HPV testing showed its superiority. The result of HCII was better than that of cytology, particularly for the sensitivity (90\%) and NNP (99\%), even better than those (sensitivity $62 \%$ and NPV 96\%) of combining the two assays. We analyze: (1) at the beginning of two FU visits, one patient was diagnosed as the residual/ recurrent disease in each time, however, the rate of HR-HPV DNA clearance was only $87 \%$ in the second FU visit; (2) in the two final FU visits, the $23(23 / 25,92 \%)$ patients identified as the residual/recurrence disease corresponded to the result of Milojkovic and Bonder (Milojkovic et al., 2002; Bonder et al., 2002) which showed the most residual/recurrent diseases occurring within a 30-month period after treatment. Among them, 4 cases showing the persistence of HR-HPV DNA+/ cytology during the FU were histologically confirmed as the adenocarcinoma in situ or the epithelioid carcinoma in situ at the end of FU, and this observation, as reported by Zielinski et al. (2003), demonstrating the power of HPV testing for monitoring women after treated for CIN II-III; on the other hand, of 3 cases showing cytology+/ HR-HPV DNA- diagnosed as the residual/recurrent disease at the end of FU visit, one was CIN I and the other two were carcinoma in situ and 
micro-invasive, respectively. Other studies have reported similar experiences, in which false-negative results of HRHPV have not been rare (Cuzick et al., 1999; Rietmuller et al., 1999; Zielinski et al., 2003; Kulmala et al., 2004). This phenomenon provokes that if HPV DNA detection is used only as the screening method, the false-negative results of HR-HPV would truly be a clinical problem. In this case, the combination of the two assays is the best method for monitoring patients having been treated for CIN II-III, though its sensitivity and NPV can't reach to $100 \%$.

Residual /or relapse is the major concern in conservative treatment of high grade CIN. In the present study, the overall 25 patients $(25 / 158,15.8 \%)$ histologically being confirmed as the residual/recurrent disease, of which, $9(9 / 25,36 \%)$ cases were verified the negative margin, and such result was similar to the data reported by other authors who had pointed out that the recurrence rate ranges from $2 \%$ to $38 \%$ in patients with clean margins (Jakus et al., 2001; Kang et al., 2001; Costa et al., 2003). Accordingly, the reasons for the residual/ recurrence after LEEP might be (i) multifocal disease; (ii) inadequate examination of surgical specimens or (iii) HR-HPV DNA persistence (Nagai et al., 2000; Costa et al., 2003; Sarian et al., 2004). In reality, all those reasons involving the correlation among the persistence HR-HPV DAN are the positive margin and the result of cytology. What our results support the failure of the original primary treatment is generally the incomplete removal of CIN lesions (Nagai et al., 2000), which was conducted finally, as shown in Table 2, the residual/recurrent disease because of the persistence of the lesion and HR-HPV DNA. As for women with negative margin, it is acceptable to say that the reason resulting in the residual/recurrent disease in the present study is the multifocal disease and the persistence HR-HPV DNA. The data collected also indicates that none of the women is identified the residual/recurrent disease if HR-HPV DNA/cytology persists negative after treatment, and is not related to the margin. It is concluded that the margin should depend on the persistence HPV and cytology once it becomes one of the predictors of the residual/recurrent disease.

Most of the large-scale clinicoepidemiologic studies have established that HR-HPV is closely associated with the pathogenesis of cervical dyplasias and carcinomas (Munoz et al., 1992; Bosch et al., 1995). Some researchers reported HPV infection in the epithelium adjacent to cervical neoplastic lesions and in exfoliated cells from the cytologically normal cervix, indicating that widespread infection with virus occurring in association with normal genital mucosa (Macnab et al., 1986; Colgan et al., 1989). In the current study, after treatment, the majority of women always showed the persistence HR-HPV DNA earlier than the abnormal cytology, all of which were finally confirmed as the CIN (Figure 1 and Table 3). All cases indicated that using HPV testing in early FU visit was the key to finding out the residual/recurrence disease. In this study, as far as at the 6th month FU, the residual/recurrent disease was found only in $8 \%$ of the research objects, while $92 \%$ were identified at the two final FU visits, i.e. at the 12th month and 18th month FU visit. Moreover, there was almost no difference in the sensitivity and NNP between at the 3rd month and at the 6th month FU visit (Figure 1). For this reason, the present research supports the ideas put forward by Zielinski and Amiram (Zielinski et al., 2003; Amiram et al., 2003): the FU visit beginning at the 6th month after treatment and lasting at least 24 months with the combination of cytology and HPV testing is reasonable.

The advantage of HCII is that it can either detect HRHPV or measure the viral load (Pretet et al., 2004). It is found that there was a linear relationship between the women with pre-treatment viral load above 500 RLU and the risk of the residual/recurrent disease, compared with those of inferior to 500 RLU as reported by Song (Song et al., 2005). Unfortunately, investigators highlighted a potential role of HPV in predicting the size and the severity of cervical lesions, as well as the presence of occult lesions which were not accessible by clinical examination. In this case, as suggested by Alonso et al. (2006), again emphasis is laid on that the clinician should pay attention to women with pre-treatment HR-HPV load. In contrast, no relationship was observed between women with pro-treatment viral load and the risk of the residual/ recurrent disease.

Based on the results of this study, it could come to conclusion that: $(i)$ the persistence HR-HPV DNA is the root cause of the residual/recurrent disease for the women treated for high-grade CIN; the pre-treatment viral load and margin can be seen as the predictor; (ii) the FU visit beginning at the 6th month post-treatment and lasting at least 24 months with the combination cytology and HPV testing; (iii) patients with high pre-treatment HPV load, which is considered as one risk of developing the residual/ recurrent disease, more attention (especially above 500 RUL/PC) should be paid by clinicians.

\section{Acknowledgements}

The study was supported by Guangxi Natural Science Foundation Projects (2013GXNSFAA019254)

\section{References}

Alonso I, Torne A , Puig-Tintore LM, et al (2006). Pre- and post-conization high-risk HPV testing predicts residual/ recurrent disease in patients treated for CIN 2-3. Gynecol Oncol, 103, 631-6.

Bar-Am A, Gamzu R, Levin I, et al (2003). Follow-up by combined cytology and human papillomavirus testing for patients post-cone biopsy: results of a long-term follow-up. Gynecol Oncol, 91, 149-53.

Barron BA, Fischetti VA, Zabriskie JB (1970). Studies of the bacteriophage kinetics of multicellular systems: a statistical model for the estimation of burst size per cell in streptococci. $J$ Appl Bacteriol, 33, 436-42.

Bosch X, Manos MM, Munoz N, et al (1995). Prevalence of human papillomavirus in cervical cancer : a woldwide perspective. J Natl Cancer Inst, 87, 796-802.

Colgan TJ,Percy ME, Suri M, et al (1989). Human papillomavirus infection of morphologically normal cervical epithelium adjacent to squamous dysplasia and invasive carcinoma. Hum Patho, 20, 316-9.

Costa S, De Simone P, Venturoli S, et al (2003). Factors predicting human papillomavirus clearance in cervical intraepithelial neoplasia lesions treated by conization. 
Gynecol Oncol, 90, 358-65.

Cuzick J, Beverley E, Ho L, et al (1999). HPV testing in primary screening of older women. Br J Cancer, 81, 554-8.

Dalstein V, Riethmuller D, Sautiere JL, et al (2005). Detection of cervical precancer and cancer in a hospital population; benefits of testing for human papillomavirus. Eur J Cancer, 40, 1225-32.

Du LB, Li HZ, Wang XH, et al (2014). Analysis of cancer incidence in Zhejiang cancer registry in China during 2000 to 2009. Asian Pac J Cancer Prev, 15, 5839-43.

Felix JC, Muderspach LI, Duggan BD, et al (1994). The significance of positive margins in loop electrosurgical cone biopsies. Obstet Gynecol, 84, 996-1000.

Flannelly G, Bolger B, Fawzi H, et al (2001). Follow up after LLETZ: could schedules be modified according to risk of recurrence? BJOG, 108, 1025-30.

Gardeil F, Turner MJ (1997). A study of treatment failures following large loop excision of the transformation zone for the treatment of cervical intraepithelial neoplasia. $\mathrm{Br} \mathrm{J}$ Obstet Gynaecol, 104, 1325.

Hanau CA, Bibbo M (1997). The case for cytologic follow-up after LEEP. Acta Cytol, 41, 731-6.

Hoskins WJ, Bundy BN, Thigpen JT, et al (1992). The influence of cytoreductive surgery on recurrence-free interval and survival in small-volume stage III epithelial ovarian cancer: a gynecologic oncology group study. Gynecol Oncol, 47, 159-66.

Houfflin Debarge V, Collinet P, Vinatier D, et al (2003). Value of human papillomavirus testing after conization by loop electrosurgical excision for high-grade squamous intraepithelial lesions. Gynecol Oncol, 90, 587-92

Jakus S, Edmonds PDunton CKing SA (2000). Margin status and excision of cervical intraepithelial neoplasia: a review. Obstet Gynecol Surv, 55, 520-7.

Kang SB, Roh JW, Kim JW, et al (2001). A comparison of the therapeutic efficacies of large loop excision of the transformation zone and hysterectomy for the treatment of cervical intraepithelial neoplasia III. Int J Gynecol Cancer, 11, 387-91.

Kulmala SM, Kulmala SM, Syrjanen S, et al (2004). Human papillomavirus testing with the hybrid capture 2 assay and PCR as screening tools. J Clin Microbiol, 42, 2470-5.

Li PL, Tan HZ (2015). Expression of PPAR $\gamma$, p27 and Ki67 in cervical cancer and its clinical significance. $J$ Int Transl Med, 3, 513-9.

Macnab JC, Walkinshaw SA, Cordiner JW, et al (1986). Human papillomavirus in clinically and histologically normal tissue of patients with genital cancer. N Engl J Med, 315, 1052-8.

Meisels A, Begin R, Schneider V (1977). Dysplasias of uterine cervix: epidemiological aspects: role of age at first coitus and use of oral contraceptives. Cancer, 40, 3076-81.

Munoz N, Bosch FX, de Sanjose S, et al (1992). The causal link between human papillomavirus and invasive cervical cancer: a population-based case-control study in Colombia and Spain. Int J Cancer, 52, 743-9.

Murdoch JB, Morgan PR, Lopes A, et al (1992). Histological incomplete excision of CIN after large loop excision of the transformation zone (LLETZ) merits careful follow up, not retreatment. Br J Obstet Gynaecol, 99, 990-3.

Nagai Y, Maehama T, Asato T, et al (2000). Persistence of human papillomavirus infection after therapeutic conization for CIN 3: is it an alarm for disease recurrence? Gynecol Oncol, 79, 294-9.

Nobbenhuis MA, Meijer CJ, van den Brule AJ, et al (2001). Addition of high-risk HPV testing improves the current guidelines on follow-up after treatment for cervical intraepithelial neoplasia. Br J Cancer, 84, 796-801.
Paraskevaidis E, Koliopoulos G, Malamou-Mitsi V, et al (2001). Large loop excision of the transformation zone for treating cervical intraepithelial neoplasia: a 12-year experience. Anticancer Res, 21, 3097-9.

Pinto AP, Crum CP (2000). Natural history of cervical neoplasia: defining progression and its consequence. Clin Obstet Gynecol, 43, 352-62.

Prendiville W, Cullimore J, Norman S (1989). Large loop excision of the transformation zone (LLETZ). A new method of management for women with cervical intraepithelial neoplasia. Br J Obstet Gynaecol, 96, 1054-60.

Pretet JL, Dalstein V, Monnier-Benoit S, et al (2004). High risk HPV load estimated by Hybrid Capture II correlates with HPV 16 load measured by real-time PCR in cervical smears of HPV16-infected women. J Clin Virol, 31, 140-7.

Quinn M, Babb P, Jones J, et al (1999). Effect of screening on incidence of and mortality from cancer of cervix in England: evaluation based on routinely collected statistics. $B M J$, 318, 904-8.

Riethmuller D, Gay C, Bertrand X, et al (1999). Genital human papillomavirus infection among women recruited for routine cervical cancer screening or for colposcopy determined by Hybrid Capture II and polymerase chain reaction. Diagn Mol Pathol, 8, 157-64.

Sarian LO, Derchain SF, Andrade LA, et al (2004). HPV DNA test and Pap smear in detection of residual and recurrent disease following loop electrosurgical excision procedure of high-grade cervical intraepithelial neoplasia. Gynecol Oncol, 94, 181-6.

Shen XR, Feng R, Chai J, et al (2014). Modeling age-specific cancer incidences using logistic growth equations: implications for data collection. Asian Pac J Cancer Prev, 15, 9731-7.

Song SH, Lee JK, Oh MJ, et al (2006). Persistent HPV infection after conization in patients with negative margins. Gynecol Oncol, 101, 418-22.

Tangtrakul S, Linasmita V, Israngura N, et al (2002). Detection of residual disease by cytology in patients with cervical intraepithelial neoplasia III post-large loop excision of the transformation zone. J Obstet Gynaecol Res, 28, 95-8.

Valasoulis G, Stasinou SM, Nasioutziki M, et al (2014). Expression of HPV-related biomarkers and grade of cervical intraepithelial lesion at treatment. Acta Obstet Gynecol Scand, 93, 194-200.

Wang Y, Yu YH, Shen K, et al (2014). Cervical cancer screening and analysis of potential risk factors in 43, 567 women in Zhongshan, China. Asian Pac J Cancer Prev, 15, 671-6.

Zhang B,Zhou AF, Zhu CC, et al (2014). Risk factors for cervical cancer in rural areas of Wuhan China: a matched case-control study. Asian Pac J Cancer Prev, 14, 7595-600.

Zielinski GD, Rozendaal L, Voorhorst FJ, et al (2003). HPV testing can reduce the number of follow-up visits in women treated for cervical intraepithelial neoplasia grade 3. Gynecol Oncol, 91, 67-73. 